\title{
A question of evidence
}

\author{
Theodora Bloom executive editor
}

The BMJ

A central tenet of evidence based medicine is that practice should change when new evidence comes to light. But how good does the evidence have to be?

Public Health England recently recommended that everyone over 4 years old should take a low dose of vitamin D, particularly to compensate for low sunlight in autumn and winter (doi:10.1136/i6183). But the evidence to support this recommendation is as yet weak (doi:10.1136/bmj.i6201). Side effects and the potential effect of overdose mean that this apparently benign recommendation could in fact lead to harmful overtreatment (doi:10.1136/i6183).

Any "innovative" change of practice should be based on real data, ideally from large scale randomised controlled trials and not from one small scale pilot study, says Margaret McCartney (doi:10.1136/bmj.i6108). But how reliable is the randomisation in such trials? Laura Clark and colleagues (doi:10.1136/bmj. i5663) have rerun a 2002 analysis of randomisation methods and find no improvement. They conclude that funders, researchers, and journals must all do better in tackling this key methodological problem.
Nicola Veronese and colleagues used very large scale cohort data to investigate the well known $\mathrm{U}$ shaped association between body mass index and mortality (doi:10.1136/bmj.i5855). By including up to four "healthy lifestyle" factors-encompassing diet, alcohol intake, smoking, and exercise - the U became a straight line, with the lowest all cause death rates among people who combine low BMI with a healthy lifestyle. The authors speculate that previous studies failed to account for people with a low BMI as a result of ill health.

The surgeon David Sellu's conviction for manslaughter was quashed recently by the Court of Appeal-but not as a result of new evidence. Clare Dyer reports that the judge in the original trial was deemed not to have briefed the jury adequately on how to distinguish "gross negligence" (a crime) from simple negligence (doi:10.1136/bmj.i6274). Liam Donaldson asks whether any health professional should be charged with manslaughter if there is no suggestion of reckless or wilful misconduct (doi:10.1136/bmj.i6268). Usually, numerous systemic failures contribute to any case of avoidable death, and pinning the blame on one person is not the best route to patient safety. 